\title{
Urethral stricture after phalloplasty
}

\author{
Min Suk Jun ${ }^{1}$, Richard A. Santucci ${ }^{2}$ \\ ${ }^{1}$ Reconstructive Urology and Trauma, Temple University Hospital, Philadelphia, PA, USA; ${ }^{2}$ Crane Surgical Services, Austin, TX, USA \\ Contributions: (I) Conception and design: All authors; (II) Administrative support: All authors; (III) Provision of study materials or patients: All \\ authors; (IV) Collection and assembly of data: All authors; (V) Data analysis and interpretation: All authors; (VI) Manuscript writing: All authors; (VII) \\ Final approval of manuscript: All authors. \\ Correspondence to: Min Suk Jun, DO, MS. Department of Urology, Temple University Hospital, Suite 330 Parkinson Pavilion, Zone C, Philadelphia, \\ PA 19140, USA. Email: minsukjun@gmail.com.
}

\begin{abstract}
Phalloplasty is a critical step for many transgender men who seek relief from gender dysphoria; however, phalloplasty is a difficult and complex surgery with many potential complications. The most common complications are urinary, mostly comprised of urethrocutaneous (UC) fistulas and urethral strictures. Improvements in surgical technique have driven down complication rates over the past few decades. Despite these innovations, complication rates remain high, and transgender surgeons must be well versed in their diagnosis and treatment. Over the same time period, gender affirming surgery has seen unprecedented growth in the United States. Transgender surgeons are few, and their patients often travel great distances for their index surgery. As such, locally available reconstructive urologists will be called upon to treat these complications with greater frequency and must be proficient in diagnosis and treatment to help these patients achieve a good outcome.
\end{abstract}

Keywords: Gender confirming surgery; gender dysphoria; phalloplasty; urinary fistula; urethral stricture; transgender

Submitted Nov 15, 2018. Accepted for publication May 20, 2019.

doi: 10.21037/tau.2019.05.08

View this article at: http://dx.doi.org/10.21037/tau.2019.05.08

\section{Introduction to phalloplasty}

The requirements of an ideal phalloplasty as described by Hage and Graaf are: (I) a one-stage procedure; (II) producing an aesthetically acceptable neophallus with (III) a neourethra that allows for voiding while standing; (IV) enough bulk to tolerate a prosthetic stiffener; (V) adequate tactile and erogenous sensation; and (VI) reproducible results with (VII) minimal scarring and functional loss of the donor site (1). A modern revision of these requirements should note that strict adherence to these tenets should not supersede the overarching mission to minimize complication rates and provide patient satisfaction. Be it standing to void or penetrative intercourse, each individual will have a personal preference, and the surgeon should tailor the phalloplasty to meet these needs. Furthermore, if a two-stage procedure will clearly produce a superior result, then one should strive for that.
The most common complications of phalloplasty are fistulas and strictures, resultant from the fact that delicate flaps are used to create a long urethral extension to the natal female urethra. The neophallus urethra is comprised of four regions: (I) the native urethra; (II) fixed urethra (pars fixa); (III) pendulous urethra (pars pendulous), and 4) urethral meatus (Figure 1A,B). The junction between the fixed urethra and pendulous urethra, often called the anastomotic urethra, tends to be the region in which many phalloplasty complications arise. A large review of 665 patients drawn from 11 studies revealed that an average of 0.51 strictures and/or fistulas can be expected per free forearm flap phalloplasty (2). This has fallen to as low as $22 \%$ in our recent series, using optimized techniques of urethral construction (3).

Through much trial and error, radial forearm free flap (RFFF) phalloplasty has become the most popular choice amongst transgender surgeons. Other phalloplasty 

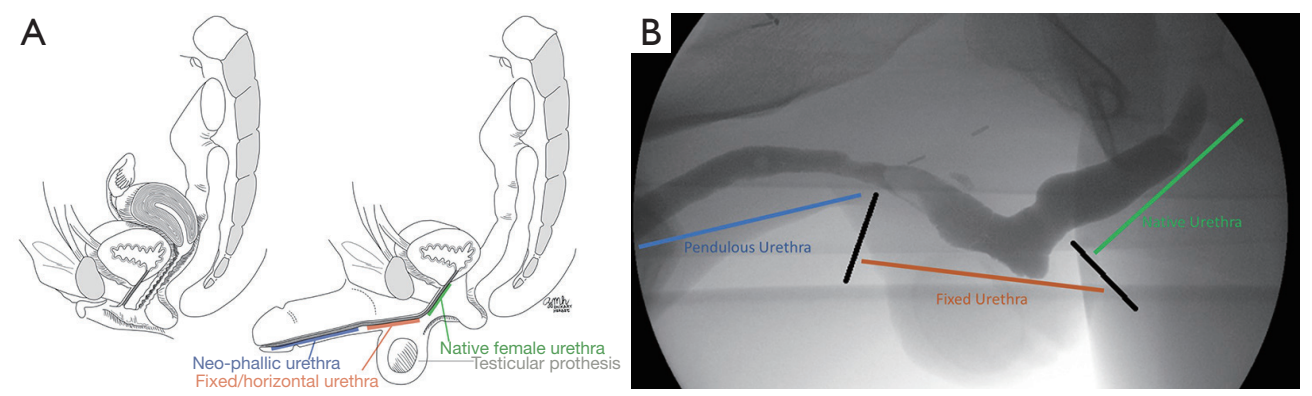

Figure 1 Neourethra anatomy. (A) Schematic of neourethra anatomy shows the native urethra, fixed urethra, and pendulous urethra; (B) retrograde urethrogram of a healthy urethra after phalloplasty shows native urethra (green), pars fixa (orange) and pars pendulous (blue).
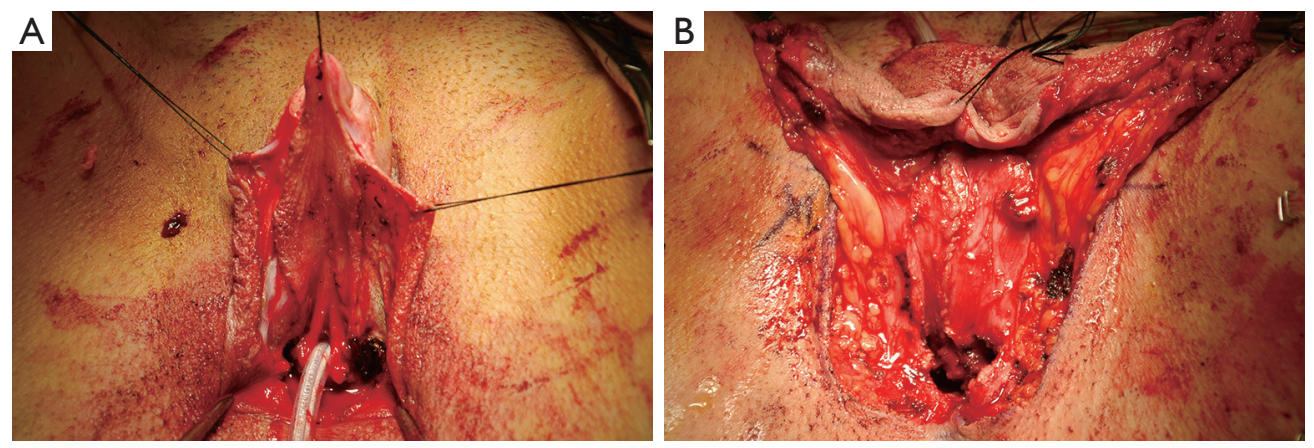

Figure 2 Anastomotic urethra anatomy. (A) The pars fixa is created from paravaginal mucosal flaps that are mobilized to midline and tubularized to create a long urethral tube. The tissue quality of these flaps is variable and dependent on patient anatomy instead of surgeon technique. (B) The pars fixa is covered with a robust second layer of bulbospongiosus muscle which is freed up laterally and closed across the midline.

techniques include the anterolateral thigh flap (pedicled or free), latissimus dorsi free flap, suprapubic pedicled flap, and osteocutaneous free flap. Recent years have seen great acceleration in outcome improvement through refinements in technique as more phalloplasties are performed. In 1988, Matti et al. reported a $60 \%$ total graft loss of their RFFF phalloplasty (4). In contrast, a 2018 study reports a neophallus partial loss of $3.4 \%$ (3). Despite these advancements, phalloplasty remains a difficult surgery and complications remain common. This review will focus on the most common urethral complications of phalloplasty.

\section{UC fistula}

UC fistula has an incidence of $10 \%$ to $64 \%$, making it the most common phalloplasty complication (3-8). Advancements in technique have driven the UC fistula rate down, evidenced in more recent studies. The UC fistula rate in the RFFF neophallus was 64\% in a 1999 study. Importantly, this study described an interesting characteristic of UC fistulas-they occur early, and many will spontaneously resolve. Thirtysix percent of those UC fistulas closed spontaneously within two months. In total, 1.55 surgeries were required to repair the rest, highlighting another point that remains valid to this day: surgical repair does not always work on the first try. By 2011, a large study of RFFF reported UC fistula as the sole urologic complication in $25 \%$ of their cases, and $66 \%$ resolved spontaneously. The latest report to date has reported a $10 \%$ UC fistula rate in patients with a minimum follow-up of six months (3).

UC fistula will most commonly occur at the anastomotic urethra. A circumferential anastomosis in a blood-flowwatershed region combines to make this area prone to urine leakage and fistulization. During primary surgery, traditional reconstructive principles apply. Particularly important is the use of as many layers of well vascularized tissue over the repair as practical. Most transmen will opt for vaginectomy, allowing one to appropriate the bulbospongiosus muscle which lines the vaginal vestibule to create one of these extra layers (Figure $2 A, B$ ). This layer is absent in the few patients 
who prefer vagina-sparing phalloplasty. Massie et al. have shown that without this important layer, the UC fistula rate increases four-fold from $14 \%$ to $56 \%$ (9).

\section{Diagnosis of UC fistula}

Most UC fistulas are obvious and straight-forward to diagnose. The chief complaint usually includes leakage from an ectopic site during voiding. Symptoms may also include dysuria, decreased force of stream, straining, dribbling, and incomplete voiding. A physical exam may show a visible fistula, often at the ventral base of neophallus, which is in close proximity to the anastomotic urethra. Signs of infection may also be present, such as induration, erythema, tenderness, or purulent drainage. As previously mentioned, early presentation after phalloplasty favors the diagnosis of UC fistula. Urethral stricture can concomitantly present with UC fistula and may even be the inciting cause of the fistula, but strictures often present later at around the 2 to 6-month mark.

Many fistulas will close spontaneously. One can often predict if this will occur. When the leak is small and tracking through a large amount of tissue, spontaneous closure is likely. If, however, the leak is large and superficial, the lack of overlying tissue makes spontaneous resolution unlikely. The tenets of fistula repair are to create a tensionless and well vascularized closure with multiple layers. Importantly, repair must be delayed at least six months from the index surgery to allow for full healing. We typically plan for curative surgery without further diagnostic studies; exam under anesthesia with catheterization or cystoscopy is usually all that is required to determine the anatomy precisely. If the urethra cannot accommodate a 17 French flexible cystoscope, an ureteroscope or pediatric cystoscope may be used. Rarely is a retrograde urethrogram (RUG) needed unless obstructive voiding symptoms are present.

\section{Conservative management of UC fistula}

Most urethral fistulas are evident at the first trial of void after phalloplasty. In our practice, we do a trial of void over at least two days before we remove the suprapubic catheter. While some transgender surgeons prefer to keep the urethral catheter up to 30 days as conservative management of UC fistula, others believe that prolonged catherization is not helpful. For example, the previously described large and superficial UC fistula will never heal with conservative treatment, and definitive surgery will always or nearly always be required. The small fistula with much overlying tissue, on the other hand, often heals spontaneously with or without any urinary diversion. Ascha et al. reported that when fistulas heal spontaneously, they will do so within three months, well within the required six months of healing required before any surgery can be done (3). The role of urinary diversion is yet to be clearly delineated and will require more research.

\section{Surgical management of UC fistula}

The patient is placed in low dorsal lithotomy and examined under anesthesia. Flexible cystoscopy is performed. These steps are almost always sufficient to understand the anatomy fully. Closure is performed in multiple nonoverlapping layers. Well-vascularized local advancement flaps are utilized if available (Figure $3 A, B, C$ ). If the fistula is directly adjacent to a urethral stricture, both should be fixed together. In these cases, we prefer to excise the fistula and urethral stricture together and perform an anastomotic urethroplasty. If the urethral stricture calls for a first stage Johanson urethroplasty, the UC fistula will be treated simply by including it in the urethrotomy. When the UC fistula and urethral stricture are not adjacent to each other, then they should be addressed individually.

\section{Urethral stricture}

The phalloplasty urethra requires local tissue flaps, long suture lines, and three separate anastomoses. These anastomoses are blood supply "watershed points" and generally have the poorest blood supply. They are generally the epicenter of stricture formation, with an incidence of $14 \%$ to $57 \%(3,5,7,8,10,11)$. When a phalloplasty urethral stricture occurs, the distribution by location is $15 \%$ in the urethral meatus (usually as the result of partial distal penile flap loss), $24 \%$ in the pendulous urethra, $41 \%$ in the anastomotic urethra, $13 \%$ in the fixed urethra, and $8 \%$ are multifocal (12). In our experience, nearly all urethral strictures will be found at the anastomotic urethra and are less than $1.5 \mathrm{~cm}$ in length. The next most common entity in our practice is distal strictures that are often a sequela of partial flap necrosis (Figure 4). The following discussion will outline our approach for these urethral strictures.

\section{Diagnosis of urethral stricture}

Patients with urethral stricture present with typical 

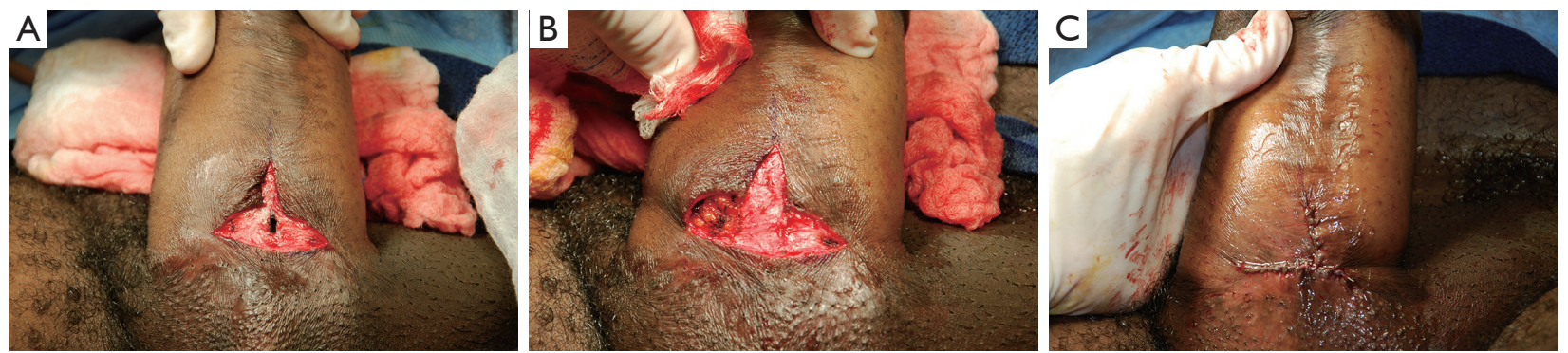

Figure 3 Urethrocutaneous fistula repair. (A) Mature urethral fistula at the base of a phalloplasty. Usually these are caused by relative necrosis of the fat over the urethra combined with fusing/healing of the urethral suture line to the phallus skin ventral suture line. A " $\mathrm{T}$ " shaped incision includes the fistula, the penile shaft suture line and the penile base suture line where it is sewn to the upper scrotum. (B) Successful primary closure of the fistula (not seen) followed by coverage with a local random tissue flap. (C) Final closure of skin over fistula repair with efforts to offset the skin suture lines with the previous fistula location/suture lines.

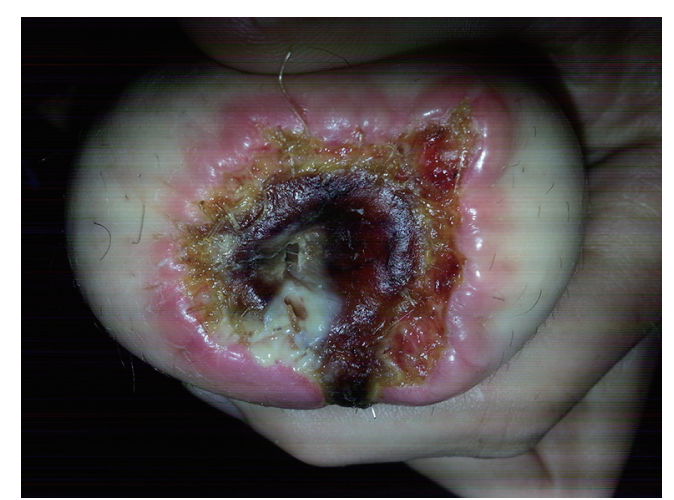

Figure 4 The edges of the flap that are used to create the phallus necessarily has the least robust blood supply as it is logically the furthest from the main vessel trunk on which the phalloplasty is based. Distal urethral necrosis or dehiscence of the meatus (connection of the urethra to the skin edges), once healed, can cause meatal stenosis.

obstructive voiding symptoms, including decreased force of stream, incomplete emptying, frequency, and urgency. Clinically suspected strictures can be confirmed in the office with flexible cystoscopy or at times by passing a urethral catheter, which will stop at the base of the penis when the common/classic "distal anastomotic" stricture is present. RUG can be used to determine stricture length, although the consistency of their presentation (short, located at the anastomosis between the pars fixa and pars pendulans) means that we often do not require RUG before surgery.

\section{Surgical management of urethral stricture}

Anastomotic urethroplasty and Johanson type urethroplasty usually with added skin grafts to widen the urethral plate is the mainstay of phalloplasty stricture treatment. Urethral dilation or direct vision internal urethrotomy is rarely helpful in the long term, although it may be used to temporize the patient before definitive surgery. In our practice, almost all anastomotic phalloplasty strictures today can be approached with anastomotic urethroplasty-either as excision and primary anastomosis (EPA) or HeinekeMikulicz urethroplasty. As in UC fistula surgery, we find it of utmost importance to delay any surgical repair until the patient is at least six months out from their index surgery. The tissue must be absent of any post-surgical inflammation, as maximum pliability and tissue integrity is required for a successful repair. To this end, if the patient presents with a urethral stricture before reaching six months after phalloplasty, one must provide urinary drainage in order to allow healing to complete with either urethral dilation, DVIU, or suprapubic catheterization. At the time of surgery, one must examine the entire urethra with cystoscopy or retrograde urethrography to rule out multifocal urethral stricture.

\section{Meatal stenosis and short urethral strictures}

The blood supply to the RFFF phalloplasty arises centrally from the flap and radiates outwards, making the distal and proximal edges most at risk to impaired perfusion. Edge necrosis can occur when severe. As a result, meatal stenosis is relatively common and make up $15 \%$ of all phalloplasty 


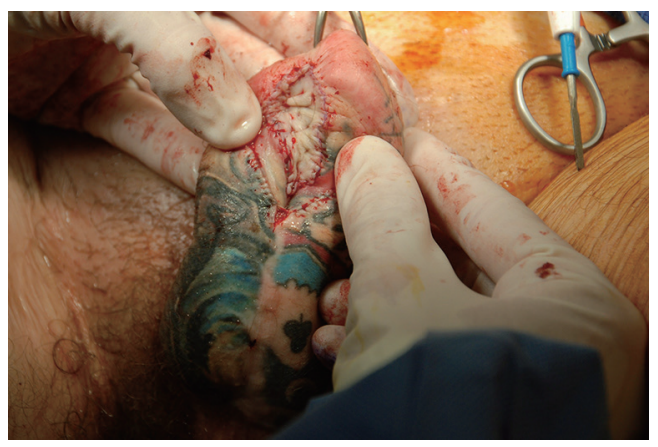

Figure 5 Distal urethral stricture after phalloplasty treated with a dorsal split thickness skin graft and single-stage urethroplasty.

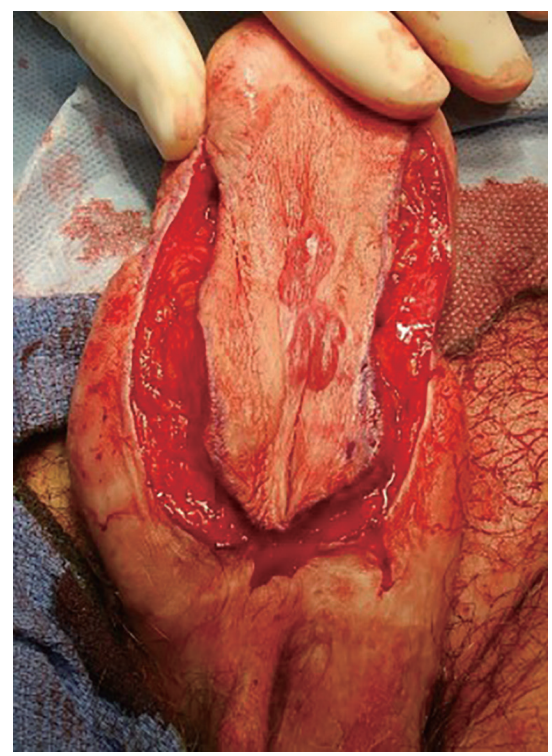

Figure 6 Second stage Johanson urethroplasty for long penile urethral stricture in an early technique "nonmicrovascular" phalloplasty. Two previously placed buccal grafts in the center have almost completely contracted. Because of this poor take of buccal grafts, we have abandoned their use in phalloplasty strictures.

urethral strictures (12). Short meatal stenosis can be easily treated with a standard meatoplasty. When the stricture is longer, we prefer a staged Johanson approach with grafting if the urethral plate requires augmentation (Figure 5). Of note, the pendulous urethra of the neophallus is composed of skin rather than urothelium; therefore, if a graft is needed, skin is harvested from the flank. Once harvested, it is thinned at the back table to split thickness and fixed in place during first stage Johanson urethroplasty. Buccal grafts are seldom or ever needed because they have a high metabolic requirement due to high cellular turnover compared to skin (13) and tend to take poorly in the already hypoperfused neophallus (Figure 6). Furthermore, while the amount of buccal mucosa is limited, flank skin can be harvested in virtually limitless amounts.

Most urethral strictures will be found at the anastomotic urethra. A nontransecting anastomotic urethroplasty may be performed when the stricture is short (approximately $1 \mathrm{~cm}$ ). Stay sutures are placed laterally and an urethrotomy is performed longitudinally. It is then closed transversely in a Heineke-Mikulicz fashion. The stay sutures will aid in performing this closure. The success rate of this method is reported at $58 \%$ (12).

When the urethral stricture is slightly longer (approximately $1.5 \mathrm{~cm}$ ) or heavily scarred, EPA is preferred. Like EPA in a natal male, a tension-free anastomosis must be made; however, mobilization of the urethra must be performed judiciously as there is no corpus spongiosum that will provide a blood supply. We favor dissecting the proximal stump more thoroughly than the distal stump. We also tend to spare the dorsal tissue if possible in an effort to minimally disrupt the blood supply to the urethra. Once we excise the strictured segment, the proximal and distal ends are spatulated and the anastomosis is completed with absorbable suture. Success rate has been reported at $57 \%$ with a mean follow-up of 34.9 months (12). Should EPA fail, repeat repair will be done with a staged Johanson urethroplasty with skin graft.

DVIU has been advocated by some in short urethral strictures as a viable option with modest success while others have reported extremely low success rate of $13 \%(14,15)$. In our practice, we have not found DVIU to be useful as a definitive strategy; its main role is as a temporizing measure to achieve urinary drainage until the patient is ready for definitive urethroplasty.

\section{Long urethral strictures}

When the stricture is longer than $2 \mathrm{~cm}$, we will opt for a staged Johanson urethroplasty. With a success rate of $70 \%$, this approach is the most durable among the urethroplasty techniques (12). The urethra is incised ventrally, moving proximally until normal-caliber urethra is encountered. Cystoscopy must be performed to ensure absence of a skip urethral stricture. The urethral plate will be widened with skin graft at the strictured segments (Figure 7). Second stage Johanson urethroplasty will be performed after 6 months (Figure 8A,B,C,D). When the stricture is particularly severe, one may forego the second stage, and the patient may elect 


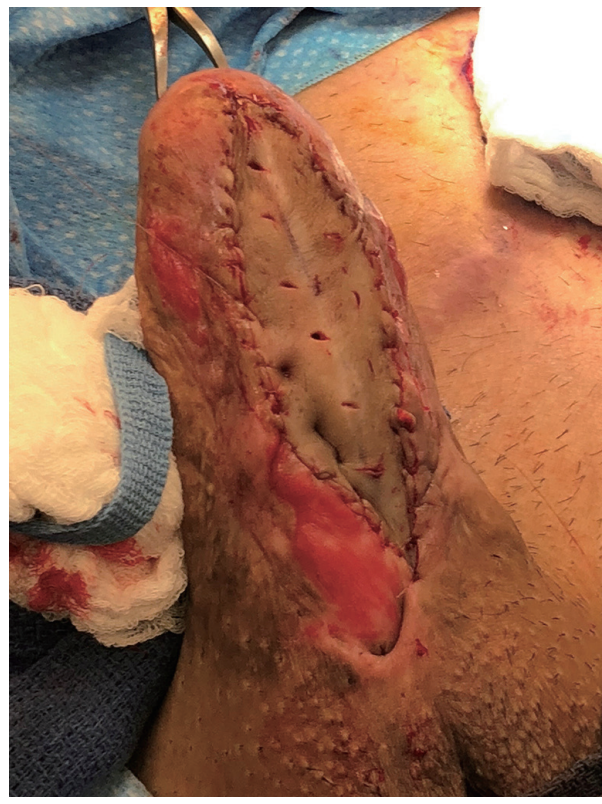

Figure 7 First stage Johanson urethroplasty of a long penile stricture with split thickness skin grafts. to void through a hypospadiac urethra or what is essentially a perineal urethrostomy if very proximal. Transmen often object to this, as standing micturition is of primary concern to these patients.

Recalcitrant urethral strictures must be referred to centers with experience in reconstructive urology and transgender medicine. Transgender surgery is an active area of research with experts continuously pushing the boundaries of surgery technique.

Extraordinarily long or recurrent strictures may require extraordinary techniques. For example, when the entire ventral neourethra is fibrotic and previous urethroplasty has failed, one may create a new groin flap to rebuild an entirely new ventral urethra, essentially creating a dualflap neophallus. Alternatively, tubularized buccal graft has been placed over fasciocutaneous labial, thigh and musculocutaneous gracilis flaps (16). These flaps should be harvested from the side contralateral to the neophallus vascular pedicle. Another special circumstance is when a urethral stricture occurs when a penile prosthesis is in situ,
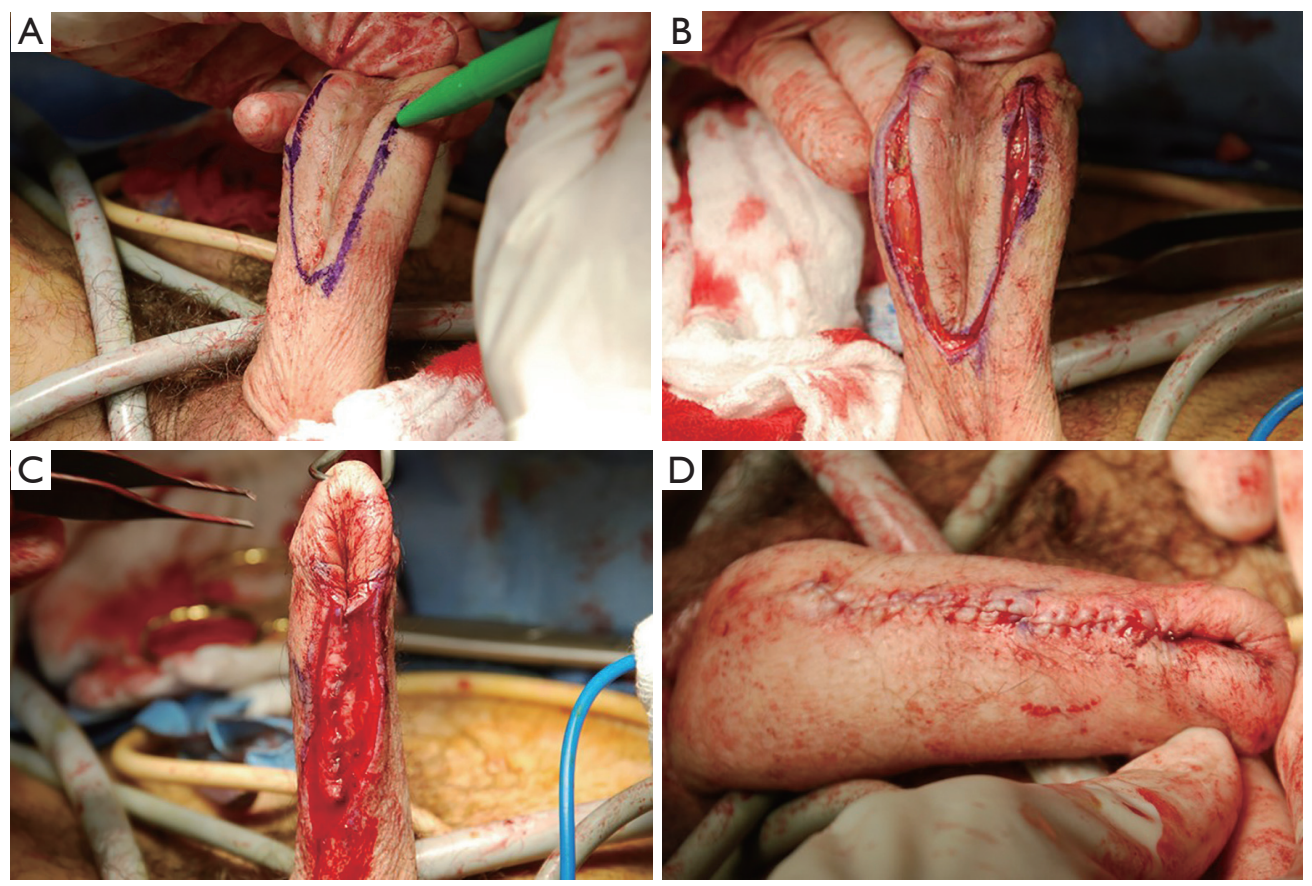

Figure 8 Second stage Johanson urethroplasty of a long penile stricture. The future urethra is marked (A) and incised (B). The inner later is tubularized (C), and the outer layer is closed (D). 
in which case extreme care must be taken with the dorsal dissection. In some cases, implant-sparing urethroplasty may not even be possible.

\section{Perineal urethrostomy}

Perineal urethrostomy is typically a measure of last resort after multiple failed attempts at urethral stricture repair. Despite its role as a salvage therapy, perineal urethrostomies are far from guaranteed with a failure rate of up to $62 \%$, highlighting just how tenuous the neourethral blood supply can be (12). The patient must accept that they will no longer be able to void while standing. The first step of the procedure is to make a long (4 to $5 \mathrm{~cm}$ ) ventral urethrotomy along the fixed urethra to account for urethral contraction. The edges are then anastomosed to the perineal skin.

\section{Conclusions}

Phalloplasty is a complex surgery associated with a $51 \%$ urethral complication rate, decreasing to $24 \%$ even in the most experienced hands. As more transgender men seek gender affirming surgery, one can expect a concomitant increase in the number of urethral complications. Reconstructive urologists will be faced with treating these urethral strictures and UC fistulas and must be well versed in the diagnosis and surgical management of these complications.

\section{Acknowledgments}

None.

\section{Footnote}

Conflicts of Interest: The authors have no conflicts of interest to declare.

\section{References}

1. Hage JJ, Graaf FHD. Addressing the ideal requirements by free flap phalloplasty: Some reflections on refinements of technique. Microsurgery 1993;14:592-8.

2. Frey JD, Poudrier G, Chiodo MV, et al. A Systematic Review of Metoidioplasty and Radial Forearm Flap Phalloplasty in Female-to-male Transgender Genital Reconstruction: Is the "Ideal" Neophallus an Achievable Goal? Plast Reconstr Surg Glob Open 2016;4:e1131.

3. Ascha M, Massie JP, Morrison SD, et al. Outcomes of
Single Stage Phalloplasty by Pedicled Anterolateral Thigh Flap versus Radial Forearm Free Flap in Gender Confirming Surgery. J Urol 2018;199:206-14.

4. Matti BA, Matthews RN, Davies DM. Phalloplasty using the free radial forearm flap. Br J Plast Surg 1988;41:160-4.

5. Fang RH, Kao YS, Ma S, et al. Phalloplasty in female-tomale transsexuals using free radial osteocutaneous flap: a series of 22 cases. Br J Plast Surg 1999;52:217-22.

6. Leriche A, Timsit MO, Morel-Journel N, et al. Long-term outcome of forearm flee-flap phalloplasty in the treatment of transsexualism. BJU Int 2008;101:1297-300.

7. Kim SK, Moon JB, Heo J, et al. A New Method of Urethroplasty for Prevention of Fistula in Female-toMale Gender Reassignment Surgery. Ann Plast Surg 2010;64:759-64.

8. Doornaert M, Hoebeke P, Ceulemans P, et al. Penile Reconstruction with the Radial Forearm Flap: An Update. Handchir Mikrochir Plast Chir 2011;43:208-14.

9. Massie JP, Morrison SD, Wilson SC, et al. Phalloplasty with Urethral Lengthening: Addition of a Vascularized Bulbospongiosus Flap from Vaginectomy Reduces Postoperative Urethral Complications. Plast Reconstr Surg 2017;140:551e-8e.

10. Rohrmann D, Jakse G. Urethroplasty in Female-to-Male Transsexuals. Eur Urol 2003;44:611-4.

11. Monstrey S, Hoebeke P, Selvaggi G, et al. Penile Reconstruction: Is the Radial Forearm Flap Really the Standard Technique? Plast Reconstr Surg 2009;124:510-8.

12. Lumen N, Monstrey S, Goessaert AS, et al. Urethroplasty for Strictures After Phallic Reconstruction: A SingleInstitution Experience. Eur Urol 2011;60:150-8.

13. Rowat JS, Squier CA. Rates of epithelial cell proliferation in the oral mucosa and skin of the tamarin monkey (Saguinus fuscicollis). J Dent Res 1986;65:1326-31.

14. Levine LA, Elterman L. Urethroplasty following total phallic reconstruction. J Urol 1998;160:378-82.

15. Lumen N, Oosterlinck W, Decaestecker K, et al. Endoscopic Incision of Short $(<3 \mathrm{~cm})$ Urethral Strictures After Phallic Reconstruction. J Endourol 2009;23:1329-32.

16. Wilson SC, Stranix JT, Khurana K, et al. Fasciocutaneous flap reinforcement of ventral onlay buccal mucosa grafts enables neophallus revision urethroplasty. Ther Adv Urol 2016;8:331-7.

Cite this article as: Jun MS, Santucci RA. Urethral stricture after phalloplasty. Transl Androl Urol 2019;8(3):266-272. doi: 10.21037/tau.2019.05.08 